\title{
Establishment and Clinical Application of the Concept of Invalid Extraction Rates in Follicular Unit Extraction
}

This article was published in the following Dove Press journal: Clinical, Cosmetic and Investigational Dermatology

\author{
Ming Lei' \\ Guang-Yuan $\mathrm{Yu}^{2}$ \\ Yong-Gang Zhao ${ }^{3}$ \\ Ji-Ping Wang' \\ Su-Yun Feng' \\ 'Center of Hair Transplantation, \\ Department of Burn and Plastic Surgery, \\ The Fourth Medical Center of the \\ General Hospital of PLA, Beijing, People's \\ Republic of China; ${ }^{2}$ Department of \\ Dermatology, The Fourth Medical Center \\ of the General Hospital of PLA, Beijing, \\ People's Republic of China; ${ }^{3}$ First \\ Department of Surgery, Beijing Armed \\ Police Corps Hospital, Beijing, People's \\ Republic of China
}

Correspondence: Ji-Ping Wang Center of Hair Transplantation, Department of Burn and Plastic Surgery, The Fourth Medical Center of the General Hospital of PLA, No. 5 I of Fucheng Road, Haidian District, Beijing, 100048, People's Republic of China Tel +86 10-66867304

Email wangjp693587@163.com
Objective: This study aims to establish the concept of invalid extraction rates in follicular unit extraction and evaluate its clinical value.

Methods: The present study involved 30 patients with alopecia. Three young surgeons (nominated A, B, and C) each performed follicular unit extraction on a randomly selected portion of the donor site of each patient for ten minutes. The outcomes were separately recorded and calculated, and converted to an invalid extraction rate for each surgeon using the formula, "invalid extraction rate $=1$ - successfully extracted follicular units/actually extracted units $\times 100 \%$."

Results: The follicular unit invalid extraction efficiency of each surgeon gradually declined. The average efficiency level of surgeon B was evaluated as excellent, while the levels of surgeons $\mathrm{A}$ and $\mathrm{C}$ were evaluated as good.

Conclusion: With experience, surgeons can speed up the process of follicular unit extraction and gradually increase performance quality through both extraction speed and success rate.

Keywords: follicular unit extraction, operating level, evaluation

\section{Introduction}

Follicular unit extraction (FUE) technology is a hair transplantation technology proposed by Rassman in the mid-1990s; ${ }^{1}$ it has become one of the most popular procedures in hair transplantation surgery. In this procedure, a hollow circular drill is used to directly drill out hair follicles, and bunches of hair from the occipital donor area of the patient are used to prepare hair follicle transplantation units. ${ }^{2-5}$ In order to remove the required grafts within a limited time, the surgeon performing the hair transplantation is required to drill relatively quickly. However, some clinicians focus on speed, ignoring the damage caused to patients by invalid drilling. Therefore, the authors propose the concept of invalid extraction rates and a related calculation method to emphasize minimizing damage to patients in FUE.

The calculation of invalid extraction rates was used to evaluate the performance of three young surgeons during drilling operations in FUE in the Burn and Plastic Surgery Department of the Fourth Medical Center of the General Hospital of the Chinese People's Liberation Army (PLA) from February to November 2018. This achieved encouraging advancement, backward supervision, and common progress. The details are reported below. 


\section{Subjects and Methods Clinical Data}

A total of 30 patients were enrolled in the present study. All patients were male, with ages ranging from 17 to 55 years. There were 23 patients with male alopecia and 7 patients with cicatricial alopecia. For each patient, hair was taken from the occipital area, and no severe trauma or surgical history was found in the donor area. Hair was taken from each patient through FUE drilling, and hair acquisition numbers ranged from 2051 to 4211 . Preoperative inspection revealed that the average hair density of the donor area was between 91 and 152 follicles per $\mathrm{cm}^{2}$, and hair diameter ranged from 0.053 to $0.097 \mathrm{~mm}$. The hair in the donor area was shaved to a length of $1 \mathrm{~mm}$. Each site was divided into four areas by a horizontal and a vertical line drawn through the position of the occipital carina. ${ }^{6-9}$ This study was conducted in accordance with the Declaration of Helsinki and approved by the Ethics Committee of The Fourth Medical Center of the General Hospital of the PLA. All patients provided written informed consent before enrollment in the study; informed consent forms of patients under the age of 18 were signed by their guardians.

\section{Inclusion and Exclusion Criteria}

The inclusion criteria were as follows: 1) normal results of routine preoperative blood and coagulation tests; 2) no infection or folliculitis on the scalp; and 3) patients eligible for hair transplant.

The exclusion criteria were as follows: 1) patients with infectious diseases such as hepatitis B, hepatitis C, syphilis, or AIDS; and 2) hair rotation in the extractive part of the posterior occipital area.

\section{Invalid Extraction Rate in Follicular Unit Extraction}

The invalid extraction rate in FUE measures the percentage of invalid extraction when surgeons take hair follicle units by drilling and reflects the need to reduce the degree of damage in the hair donor area. The calculation method is as follows: "invalid extraction rate in FUE $=1$ - successfully extracted follicular units/actually extracted units $\times 100 \%$."

\section{Equipment}

A folliscope hair detection analyzer, a FUEPK-7000 hair follicle drilling machine (LeadM, South Korea), and a number of

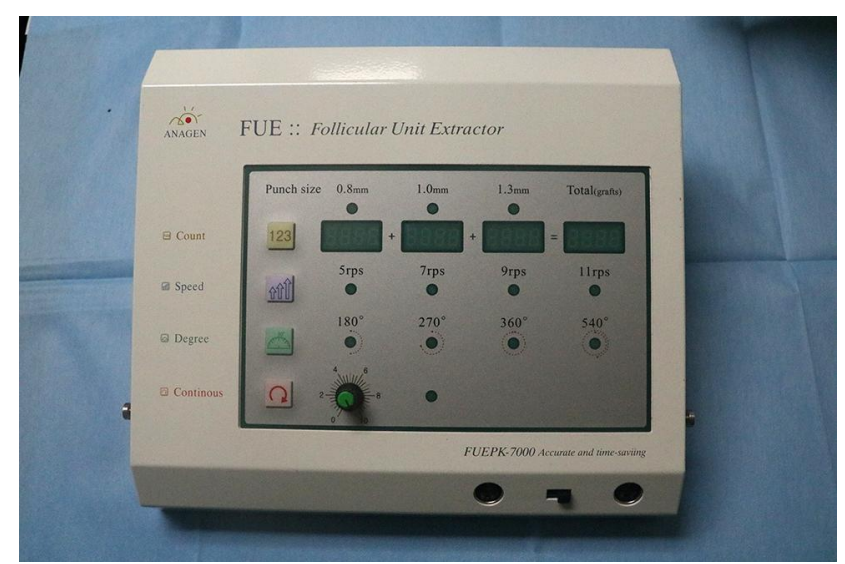

Figure I FUEPK-7000 hair follicle drilling machine.

1-mm hair extraction drills were used (Figure 1). An extraction needle with a diameter of 0.8 or $0.9 \mathrm{~mm}$ (LeadM, South Korea) was used (Figure 2), with the selection of the specific needle used based on the thickness of each patient's hair in the extraction region (Figure 3).

\section{Research Methods}

For each alopecia patient, a quarter of the donor area was randomly selected for FUE drilling by each of three young surgeons (A, B, and C). (Arrangements were made for one of the doctors to continue to extract hair from the remaining quarter, but this fourth site was not included in the statistics.) For each surgeon, the total number of hair follicles drilled and the number of hair follicles successfully drilled in ten minutes were respectively recorded. Then, the invalid extraction rate in FUE was calculated. The results were analyzed by statistical data processing. A line chart was drawn, and the development trend was observed.

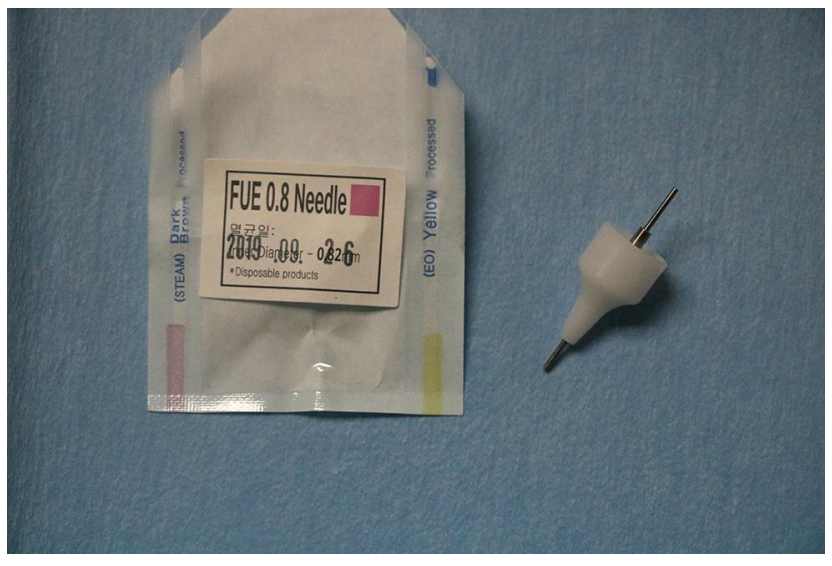

Figure $\mathbf{2}$ The drills produced by LeadM in South Korea. 


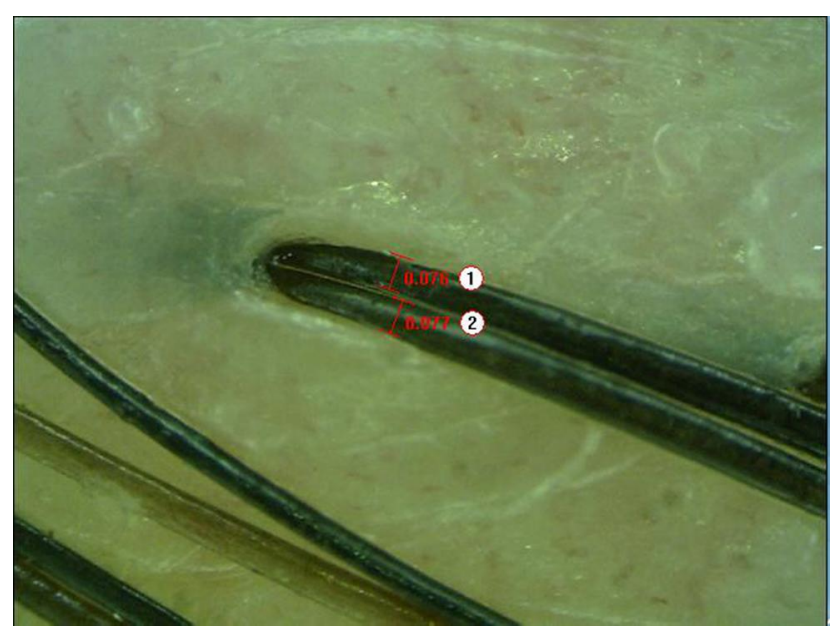

Figure 3 The diameter of posterior occipital hair follicles examined by trichoscope before operation.

\section{Evaluation Criteria}

The invalid extraction rate in FUE was classified as follows. Excellent: invalid extraction rate in FUE $\leq 15 \%$; Good: $15 \%$ <invalid extraction rate in FUE $\leq 18 \%$; Passed: $18 \%$ <invalid extraction rate in FUE $\leq 20 \%$; Failed: invalid extraction rate in FUE $>20 \%$.

\section{Results}

The following results were recorded for ten minutes of surgery. On average, surgeon A drilled 408.33 follicular units, of which 346.10 were successfully drilled; the average invalid extraction rate in FUE was therefore $15.16 \%$ (good). Surgeon B drilled an average of 408.33 follicular units, of which 351.47 were successfully drilled; the average invalid extraction rate in FUE was $13.89 \%$ (excellent). Surgeon C drilled an average of 410.17 follicular units, of which 342.57 were successfully drilled; the average invalid extraction rate in FUE was $16.37 \%$ (good).

As shown in Figure 4, the results reveal that as the experience of each of the three surgeons grew, their invalid extraction rate in FUE decreased to a certain extent. On average, surgeon B successfully extracted more follicular units (351.47) than surgeon A (346.10), and significantly more than surgeon $\mathrm{C}$ (342.57). The average invalid extraction rate in FUE of surgeon B (13.89) was correspondingly lower than that of surgeon A (15.16\%) and significantly lower than that of surgeon C (16.37\%). Surgeon $\mathrm{C}$ displayed the fastest average drilling speed but had the highest invalid extraction rates. Surgeons A and B had similar speeds, but surgeon B's invalid extraction rates were slightly lower. Surgeon B had the lowest average invalid extraction rate, which was rated as excellent, while the results for surgeons $\mathrm{A}$ and $\mathrm{C}$ were rated as good.

\section{Discussion}

Hair transplantation for androgenic alopecia has a rapid, reliable, and long-lasting effect and is a relatively ideal treatment method. ${ }^{10-12}$ Since 1939 , when Dr. Okuda in Japan discovered that hair could survive after scalp

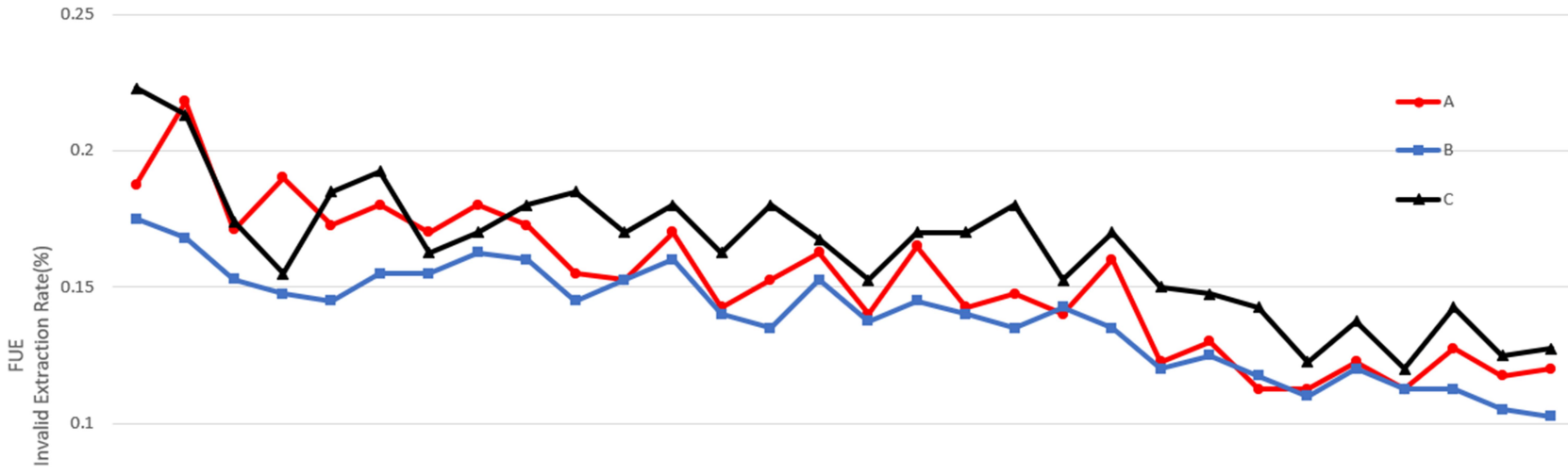

0.05

0

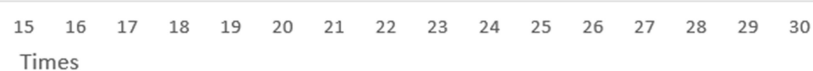

Figure 4 Change trend of invalid extraction rate of FUE by three doctors A, B and C. 
transplantation, hair transplantation technology has made revolutionary advances, beginning to mature with Rassman's proposal of FUE technology in the mid1990s. ${ }^{13-15}$ There are two conventional surgical methods: scalp strip cutting technology and FUE technology. Since scalp strip cutting technology leaves obvious scars, in clinics most patients accept FUE technology. ${ }^{16}$

Compared with traditional scalp string cutting technology, FUE has the advantages of small trauma, low cost, unconspicuous scar in the donor area, and quick recovery. The hair follicles can be implanted after separation and collection, and the surgery requires fewer operators, thereby completely overcoming the shortcomings of scalp strip cutting technology. However, FUE also has its limitations. The operation is lengthy, with the time taken for hair follicle extraction almost the same as that for implantation, and the patient needs to be placed in the prone position while the hair follicles are extracted. The prolonged period in this position can cause discomfort for those with a lumbar vertebral disease and those unaccustomed to the position. Furthermore, for hair transplantation over large areas, multiple operations are needed as the number of follicles grafted from one FUE is limited. ${ }^{17-19}$

Reducing the invalid extraction rate in FUE means reducing error rates in hair follicle drilling. This is the common wish of patients and surgeons; it is also the aim and direction of FUE technology development and the surgeon's efforts. When a surgeon first learns the technique of drilling hair follicles, they usually have a high invalid extraction rate, not only causing the nonutilization of a large quantity of drilled hair follicles but also increasing the trauma of patients and the drilling time and/or the number of operations required. However, there are currently no objective criteria for evaluating a surgeon's level of skill in this area. There are many factors that lead to hair follicle inactivation, including mechanical clamping injury, dry hair follicles, abnormal temperature, and a long time in vitro. Any of these factors may cause the failure of implanted hair follicle survival. The integrity of hair follicle extraction is the basis for the survival of transplanted hair follicles. Our study focuses on the integrity of the hair follicle during the extraction process, proposing the concept of invalid extraction rates in FUE to increase the drilling speed for hair follicle units while reducing the invalid drilling rate, thus preventing excessive damage to the hair and to the patient.

The invalid extraction rate in FUE can be used to evaluate the technical level of a surgeon in FUE drilling.
Drilling speed is one criterion for evaluation; we propose the invalid extraction rate as an additional criterion that more accurately reflects the surgeon's skills. Under the same conditions for drilling hair follicles, surgeons with low invalid extraction rates in FUE will reduce patients' pain and increase the utilization rate for hair follicles, which will reduce the damage to patients. Reducing the invalid extraction rate in FUE while improving drilling speed will be of great benefit to both patients and surgeons. The measured invalid extraction rate for a surgeon varies between patients, often correlating with the patient's hair density, hair thickness, hair growth direction, scalp hardness, and other factors. ${ }^{20}$ However, given consistent technical characteristics, the surgeon's invalid extraction rate in FUE can be expected to decrease to a certain extent with experience.

By calculating and examining the invalid extraction rate in FUE, surgeons' technical proficiency can be observed to change with the accumulation of experience. The statistical findings concerning the three surgeons in this study and their 30 patients indicate that as total drilling time increased, the invalid extraction rate in FUE for each surgeon gradually decreased. This measure can be used to evaluate whether a surgeon's level of operating efficiency has improved. Similarly, surgeons can improve their technique by understanding their invalid extraction rate in each FUE and summing up and learning from their experience.

A drilling surgeon's invalid extraction rate in FUE can therefore be used as an evaluation criterion. At present, although hair transplantation technology is maturing globally, the technical level of professional practitioners remains uneven. Management in this field is chaotic, and there are no complete or systemic technical standards. Therefore, the proposed invalid extraction rates in FUE can support hair transplantation surgery technology in China in becoming standardized and of higher quality, and contribute to the establishment of effective criteria for assessing a surgeon's technical specifications.

This study has certain limitations. First, the extraction difficulty varies greatly between patients. Because the sample size for the study was limited, there is a certain selection bias. Second, although it is (theoretically) best to compare extraction rates over the same scalp area of the same patient, it is impossible for more than one doctor to extract from the same site. We selected the two sides of the scalp of each patient for comparison, which may have influenced the results. 


\section{Funding}

Chinese Medical Association L'OREAL Chinese healthy skin/hair research project (H2014111108).

\section{Disclosure}

The authors declare that they have no conflicts of interest for this work.

\section{References}

1. Bunagan MJ, Banka N, Shapiro J. Hair transplantation update: procedural techniques, innovations, and applications. Dermatol Clin. 2013;31(1):141-153. doi:10.1016/j.det.2012.08.012

2. Konishi K, Sugimoto I, Kakizaki H, Ichinose A. Reshaping the eyebrow follicular unit transplantation from excised eyebrow in extended infrabrow excision blepharoplasty. Clin Ophthalmol. 2012;6:247-252. doi:10.2147/OPTH.S28353

3. Zhao YG, Wang JP, Lei M, Feng SY. Concept of follicular unit extraction efficiency and its clinical application. Chin J Aesthetic Plast Surg. 2014;25:422-424.

4. Williams KL Jr. Current practices and controversies in cosmetic hair restoration. Dermatol Surg. 2013;39:797-801. doi:10.1111/dsu.12163

5. Rogers NE. Hair transplantation update. Semin Cutan Med Surg. 2015;34:89-94. doi:10.12788/j.sder.2015.0131

6. Inui S, Itami S. Molecular basis of androgenetic alopecia: from androgen to paracrine mediators through dermal papilla. J Dermatol Sci. 2011;61(1):1-6. doi:10.1016/j.jdermsci.2010.10.015

7. Zhao YG, Wang JP, Lei M, Feng SY. Clinical value of preoperative estimation of the ratio of hair number to follicular-unit in the donor site for FUE operation. Chin J Aesth Plast Surg. 2015;26:162-164.

8. Raposio E, Caruana G. Scalp surgery: quantitative analysis of follicular unit growth. Plast Reconstr Surg Glob Open. 2015;3(10):e539. doi:10.1097/GOX.0000000000000524
9. Ors S, Ozkose M, Ors S. Follicular unit extraction hair transplantation with micromotor; eight years experience. Aesthetic Plast Surg. 2015;39:589-596. doi:10.1007/s00266-015-0494-8

10. Lei M, Fan JC. The related therapeutic and pathogeny study progress of androgenetic alopecia. Chin J Aesthetic Med. 2008;17:1812-1815.

11. Azar RP, Thomas AH, Lindner G. Hair follicle plasticity with complemented immune-modulation following follicular unit extraction. Int J Trichology. 2015;7:16-23. doi:10.4103/0974-7753.153451

12. Tomc CM, Malouf PJ. Eyebrow restoration: the approach, considerations, and technique in follicular unit transplantation. J Cosmet Dermatol. 2015;14(4):310-314. doi:10.1111/jocd.12170

13. Civaş E, Aksoy B, Aksoy HM, Eski M, Yucel K. Hair transplantation for therapy-resistant alopecia areata of the eyebrows: is it the right choice? J Dermatol. 2010;37(9):823-826. doi:10.1111/j.13468138.2010.00872.x

14. Azar RP, Thomas AH, Maurer M, Lindner G. Alterations in hair follicle morphology and hair shaft production after follicular unit transplantation. Am J Dermatopathol. 2016;38(10):732-738. doi:10.1097/DAD.0000000000000526

15. Marzola M. Single-Scar Harvesting Technique. Philadelpaia: Elsevier Saunders; 2006:83-85.

16. Harris JA. Follicular unit extraction. Facial Plast Surg Clin North Am. 2013;21(3):375-384. doi:10.1016/j.fsc.2013.05.002

17. Gho CG, Martino Neumann HA. Donor hair follicle preservation by partial follicular unit extraction. A method to optimize hair transplantation. $J$ Dermatolog Treat. 2010;21(6):337-349. doi:10.3109/09546630903359814

18. Yildiz H, Ercan E, Alhan D, Sezgin M. Recipient site necrosis after tumescent infiltration with adrenaline in hair transplantation. Acta Dermatovenerol Croat. 2015;23(3):233-234.

19. Onda M, Igawa HH, Inoue K, Tanino R. Novel technique of follicular unit extraction hair transplantation with a powered punching device. Dermatol Surg. 2008;34(12):1683-1688. doi:10.1111/j.15244725.2008.34346.x

20. Cole JP. An analysis of follicular punches, mechanics, and dynamics in follicular unit extraction. Facial Plast Surg Clin North Am. 2013;21:437-447. doi:10.1016/j.fsc.2013.05.009
Clinical, Cosmetic and Investigational Dermatology

\section{Publish your work in this journal}

Clinical, Cosmetic and Investigational Dermatology is an international, peer-reviewed, open access, online journal that focuses on the latest clinical and experimental research in all aspects of skin disease and cosmetic interventions. This journal is indexed on CAS
The manuscript management system is completely online and includes a very quick and fair peer-review system, which is all easy to use. Visit http://www.dovepress.com/testimonials.php to read real quotes from published authors. 